\title{
Outcome Differences by Delirium Motor Subtype in Patients with Ischemic Stroke
}

\author{
Hee Won Yang', Miji Lee ${ }^{1}$, Jong Wook Shin², Hye Seon Jeong², Jei Kim²,3, and Jeong Lan Kim ${ }^{1,4} \bowtie$ \\ ${ }^{1}$ Department of Psychiatry, Chungnam National University Hospital, Daejeon, Republic of Korea \\ ${ }^{2}$ Department of Neurology, Chungnam National University Hospital, Daejeon, Republic of Korea \\ ${ }^{3}$ Department of Neurology, School of Medicine, Chungnam National University, Daejeon, Republic of Korea \\ ${ }^{4}$ Department of Psychiatry, School of Medicine, Chungnam National University, Daejeon, Republic of Korea
}

Objective This study evaluated the outcomes of ischemic stroke patients according to delirium motor subtype.

Methods This study included patients who were admitted to the stroke unit between August 2017 and March 2019 and met the DSM-5 diagnostic criteria for delirium. Patients were assessed twice weekly throughout their delirium episodes using the Korean version of the Delirium Motor Subtype Scale (K-DMSS) and the Korean version of the Delirium Rating Scale-Revised-98 (K-DRS-98). The clinical characteristics and short-term outcomes of the patients were also assessed.

Results A total of 943 stroke patients were included; the rate of incident delirium was $10.18 \%$. Of the 95 delirium patients, 34 were classified as the hyperactive subtype, 30 as the mixed subtype, 25 as the hypoactive and six as no subtype. Among the subtype groups, the hypoactive subtype had the highest initial scores on the National Institutes of Health Stroke Scale (NIHSS; 6.72 \pm 4.75 , $p=0.02$ ) and the modified Rankin Scale ( $\mathrm{mRS} ; 3.96 \pm 1.24, \mathrm{p}<0.01)$. Additionally, the mixed and hypoactive subtypes had longer durations $(\mathrm{p}<0.01)$ and more severe symptoms of delirium $(\mathrm{p}=0.03)$ than the other motor subtypes, and the hypoactive subtype group had a significantly longer hospital stay $(36.88 \pm 27.71$ days, $\mathrm{p}<0.01)$ than the other subtype groups. After adjusting for baseline covariates in a multiple linear regression analysis, these differences remained significant.

Conclusion The present results suggest that the motor subtype of delirium is associated with different characteristics and outcomes in ischemic stroke patients.

Psychiatry Investig 2019;16(11):852-859

Key Words Ischemic stroke, Delirium, Motor subtype, Outcomes.

\section{INTRODUCTION}

Delirium is a syndrome characterized by disturbances in attention and cognition that develop over a short period and tend to fluctuate in severity during the course of a day. ${ }^{1}$ It is one of the most common in-hospital complications, ${ }^{2,3}$ occurring in approximately $20 \%$ of patients, and especially in elderly patients with high comorbidity and/or preexisting cognitive impairment. ${ }^{4}$

The incidence of delirium is higher in post-stroke patients

\footnotetext{
Received: May 14, 2019 Revised: July 16, 2019

Accepted: July 30, 2019

$\triangle$ Correspondence: Jeong Lan Kim, MD

Department of Psychiatry, Chungnam National University Hospital, 82 Munhwa-ro, Jung-gu, Daejeon 35015, Republic of Korea

Tel: +82-42-280-7280, Fax: +82-42-280-7886, E-mail: thomasign@gmail.com

(c) This is an Open Access article distributed under the terms of the Creative Commons Attribution Non-Commercial License (https://creativecommons.org/licenses/bync/4.0) which permits unrestricted non-commercial use, distribution, and reproduction in any medium, provided the original work is properly cited.
}

(13-48\%) than in patients admitted to general internal medicine wards (10-25\%). ${ }^{5-8}$ However, the incidence of delirium in patients with ischemic stroke varies widely from $2.3 \%$ to $61 \%$ across studies, perhaps due to inconsistency in the diagnostic criteria applied or the inclusion criteria for study subjects. ${ }^{9} \mathrm{~Pa}$ tients with post-stroke delirium have unfavorable outcomes, including high mortality, longer hospitalization, greater risk of dementia, and a greater degree of functional dependence after hospital discharge. ${ }^{5,10,11}$

Delirium is almost invariably complicated by disturbances of motor activity, which have been the focus of greatest interest in studies of its clinical subtypes. Lipowski ${ }^{12}$ differentiated between "hypoactive" and "hyperactive" delirium, later adding a "mixed" category that included patients who exhibited elements of both during a single episode. Patients who did not meet the criteria for any of these three subtypes were categorized as "no subtype." ${ }^{13}$ However, one study ${ }^{14}$ found only $34 \%$ concordance of delirium subtypes, categorized using four com- 
mon subtyping methods, within a single delirious population. A new scale, the Delirium Motor Subtype Scale (DMSS), was subsequently developed as a valid instrument to assess motor subtypes of delirium. ${ }^{15,16}$ Although differences in detection rates, etiology, non-motor delirium phenomenology, episode duration, management, and prognosis have been identified among motor subtypes of delirium, ${ }^{17}$ these findings have been inconsistent across studies, ${ }^{18-20}$ largely due to differences in subtype definitions and patient populations. The hypoactive delirium subtype predicts a worse prognosis, with a higher risk of pressure sores and infections, whereas the hyperactive delirium subtype can be diagnosed and treated earlier due to its easily recognized features, thus improving outcomes. ${ }^{17,21}$ However, apart from these results, only a few studies ${ }^{22,23}$ have evaluated the association of delirium motor subtypes with clinical characteristics and outcomes in patients with ischemic stroke.

Therefore, the aim of this study was to evaluate clinical characteristics and outcomes according to delirium motor subtypes in patients with ischemic stroke admitted to the stroke unit (SU) of a tertiary referral hospital.

\section{METHODS}

\section{Subjects}

The study population was drawn from patients with cerebral infarction who developed delirium after admission to the SU of Chungnam National University Hospital, Daejeon, Korea, between August 2017 and March 2019. Because patients with global aphasia, sensory aphasia, and coma could not be appropriately assessed for delirium, we excluded them from the study. We also excluded patients who were admitted to the intensive care unit (ICU) and those who refused to participate. The procedures and rationale for this study, as well as the right to withdraw, were fully explained to all patients and their family members. Due to the nature of the study, informed consent was obtained from patients when possible, and proxy consent from caregivers when necessary. The study protocol was approved by the Institutional Review Board (IRB No: 2017-07-058).

\section{Study procedures}

\section{Screening procedures}

Patients were screened for delirium every day beginning on the day of SU admission. Screening was performed at the same time every day (4 p.m.) by trained SU nurses, and the Confusion Assessment Method (CAM) was used for the delirium screening. The SU nurses were trained to perform the CAM by a psychiatrist (the first author of the present study) prior to the first registration.

\section{Delirium assessment}

Patients who met the CAM criteria for delirium were assessed by a trained psychiatrist within $24 \mathrm{~h}$. Diagnosis was based on the Diagnostic and Statistical Manual of Mental Disorders (DSM)-5 criteria for delirium. The severity of delirium symptoms was assessed using the Korean version of the Delirium Rating Scale-Revised-98 (K-DRS-98). The Korean version of the DMSS (K-DMSS) was also applied to classify delirium motor subtypes.

\section{Follow-up assessments}

After the initial assessment, the presence of delirium was assessed daily and symptom severity and delirium motor subtype were reassessed twice weekly by the psychiatrist who performed the initial assessment for up to 3 weeks, or until the delirium resolved or until the patient died. This frequency of assessments allowed for consistency in the time frame between assessments, sustained observations of the motor activity profile, and the minimization of any tendency toward missing or incomplete data. For patients who were discharged with delirium, outcomes such as duration of delirium and subsequent institutionalization were evaluated through follow-up calls and visits.

\section{Assessment of predisposing factors}

Data were collected regarding demographic factors and comorbidities, including hypertension, diabetes mellitus, dyslipidemia, renal failure, hepatic failure, atrial fibrillation, ischemic heart disease, cardiac valve disease, respiratory system disorder, thyroid disease, urinary tract infection, Parkinson's disease, previous stroke history, comorbid cognitive impairment, and past delirium history. All medications prescribed to a patient during hospital admission were documented and are presented as the number of medications received when delirium occurred. Auditory and visual impairments, laboratory test results, malnutrition, urinary catheterization, physical restraints, and endotracheal intubation during hospitalization were also recorded.

\section{Outcome measures}

Outcome measures included the length of hospital stay, death during hospitalization, institutionalization upon discharge, duration of delirium, and delirium severity. The days with DSM-5 delirium were counted to determine the duration of delirium. Delirium severity was measured according to the average K-DRS-98 total severity score and the highest K-DRS-98 total severity score (items 1-13) during delirium. ${ }^{24}$ The relationship between motor subtypes and delirium outcomes was evaluated. 


\section{Assessment tools}

\section{CAM}

The $\mathrm{CAM}^{25}$ is one of the most widely used screening instruments for delirium. This measure was designed to allow nonpsychiatric clinicians to quickly diagnose delirium by assessing the presence, severity, and fluctuation of the following nine delirium features: acute onset, inattention, disorganized thinking, altered level of consciousness, disorientation, memory impairment, perceptual disturbances, psychomotor agitation or retardation, and altered sleep-wake cycle. Its diagnostic algorithm is based on four key components: 1) acute onset and fluctuating course, 2) inattention, 3) disorganized thinking, and 4) altered level of consciousness. A diagnosis of delirium according to the CAM requires the presence of features 1,2 , and either 3 or 4 . CAM has a sensitivity of $82 \%$ and a specificity of $99 \%$ in geriatric, postoperative, and intensivecare patients, but it has not been validated for stroke patients. ${ }^{26}$

\section{K-DRS-98}

The Delirium Rating Scale-Revised-98 (DRS-R98) ${ }^{24}$ is designed for the broad phenomenological assessment of delirium. The Korean version of the DRS-R98 was validated and standardized by Lim et al. ${ }^{27}$ It is a 16-item scale comprised of 13 items that assess severity and three diagnostic items with high interrater reliability, sensitivity, and specificity for detecting delirium in various neuropsychiatric populations and other inpatients. ${ }^{24}$ Each item is rated from 0 (absent) to 2 or 3 (severe), and the severity scale scores range from 0 to 39 , such that higher scores are indicative of more severe delirium.

\section{K-DMSS}

The Delirium Motor Subtype Scale (DMSS) ${ }^{15}$ is an 11-item scale used to classify delirium into several motoric subtypes. The Korean version of the DMSS was validated and standardized by Kim et al. ${ }^{28}$ Each item is rated as "absent" (score=0) or "present" (score=1). The hyperactive subtype is assigned if the patient shows at least two of the following symptoms over the previous $24 \mathrm{~h}$ : 1) increased amount of motor activity, 2) loss of control of motor activity, 3) restlessness, and 4) wandering. The hypoactive subtype is assigned if the patient shows at least two of the following symptoms over the previous $24 \mathrm{~h}$ : 1) decreased amount of activity, 2) decreased speed of actions, 3) reduced awareness of surroundings, 4) decreased amount of speech, 5) decreased speed of speech, 6) listlessness, and 7) reduced alertness/withdrawal. The mixed subtype is assigned if the patient shows both hyperactive and hypoactive symptoms. No subtype is assigned if there is no evidence of either the hyperactive or hypoactive subtype.

\section{Other stroke-related assessment tools}

At admission, the etiology of ischemic stroke was classified as large artery atherosclerosis (LAA), cardioembolism (CE), small vessel occlusion (SVO), or other, according to the Trial of Org 10172 in Acute Stroke Treatment (TOAST) criteria. ${ }^{29}$ The severity of the clinical deficit was graded using the National Institutes of Health Stroke Scale (NIHSS), a graded neurological examination that assesses consciousness, eye movements, visual field, motor and sensory impairments, ataxia, speech, cognition, and inattention. ${ }^{30}$ Motor functions were assessed using the modified Rankin Scale (mRS), a commonly used scale for measuring the degree of disability or dependence in daily activities among people who have suffered a stroke or neurological disability from other causes. ${ }^{31}$ In addition, data regarding laterality and location of the stroke lesion, stroke symptoms, and stroke complications were collected.

\section{Statistical analysis}

Demographic and clinical data are expressed as mean \pm standard deviation. Associations between continuous variables (e.g., age, length of hospital stay, duration of delirium, mean K-DRS-98 score) and delirium motor subtypes were analyzed using the Kruskal-Wallis test. Associations between categorical variables (e.g., sex, institutionalization, death during hospitalization) and delirium motor subtypes were analyzed using chi-square tests. The associations between motor subtype of delirium and outcomes, including duration of delirium, highest K-DRS-98 severity score, length of stay, NIHSS score at discharge, and mRS score at discharge, were adjusted for specific covariates that were significantly associated with the motor subtypes of delirium using a multiple linear regression analysis. All statistical analyses were conducted using SPSS software (ver. 22.0; IBM Corp., Armonk, NY, USA), and statistical significance was accepted at $\mathrm{p}<0.05$.

\section{RESULTS}

\section{Study participants}

A total of 1279 patients were admitted to the SU during the study period; Of these patients, 118 were excluded due to admission to the ICU, global aphasia, sensory aphasia, and/or coma, and 218 did not provide informed consent. Thus, 943 patients were ultimately included in the delirium cohort; the rate of incident delirium was 10.18\% (96 of 943 patients).

Initially, the present study aimed to evaluate the outcomes of these 96 delirium patients by the motor subtype, but one of the patients was discharged with unresolved delirium and could not be contacted. Thus, 95 patients were included in the final analyses (Figure 1).

Table 1 shows the clinical and demographic information of 


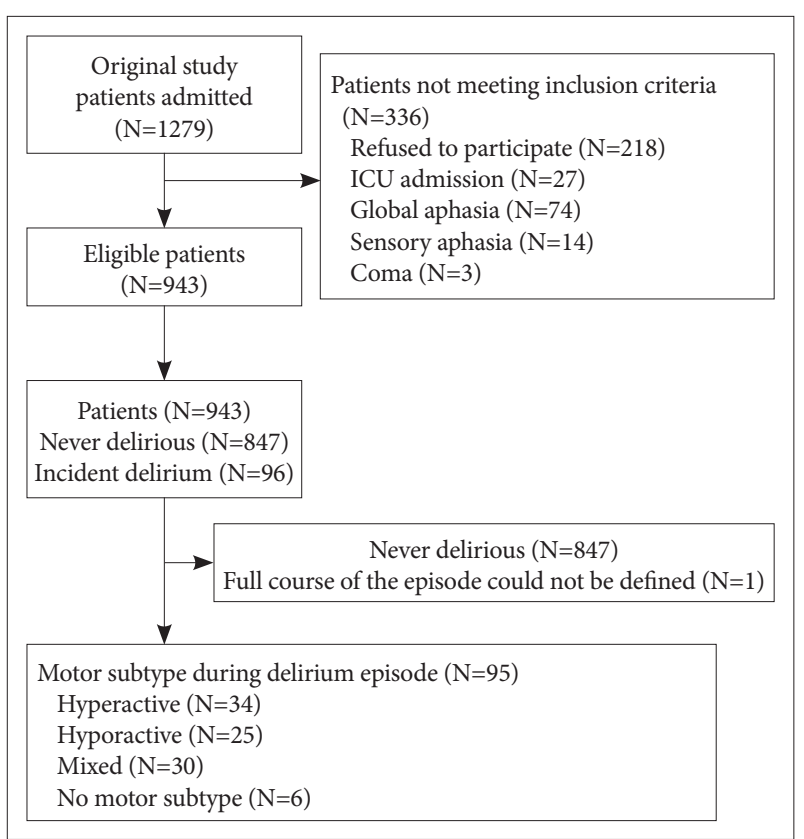

Figure 1. Flow diagram of the study.

the patients. The hyperactive subtype was identified in 34 (35.8\%) patients, the hypoactive subtype in 25 patients (26.3\%), the mixed subtype in 30 patients (31.6\%), and no subtype in six patients $(6.3 \%)$. The average number of medications received over the course of the delirium episode was $5.81 \pm 3.13$, and 17 patients (17.9\%) had comorbid cognitive impairments, such as dementia or mild cognitive impairment. The mean duration of delirium was $7.10 \pm 6.36$ days, and the mean KDRS-98 severity score over the course of delirium was 18.39 \pm 4.13 .

\section{Clinical characteristics of the motor subtype groups}

Table 2 shows the general clinical characteristics of the patients in each motor subtype group. The blood urea nitrogen (BUN) levels significantly differed according to the motor subtype ( $\mathrm{p}=0.03)$, such that the mixed subtype $(21.05 \pm 8.53)$ had a significantly higher level than the other subtype groups. There were no significant differences in terms of sex, age, laboratory test results, numbers of medications received, rates of urinary catheterization, physical restraint use, auditory and visual impairment, comorbid cognitive impairment, or past delirium history, between motor subtype groups.

Table 2 also shows the stroke-specific characteristics of the patients with delirium. Although right-sided lesions were more common than left-sided and bilateral lesions across all motor subtype groups, these differences were not statistically significant ( $\mathrm{p}=0.13)$. The NIHSS grade of stroke severity on admission was significantly higher in the hypoactive subtype group (6.72 \pm 4.75$)$ than in the other subtype groups $(\mathrm{p}=0.02)$. In ad-
Table 1. Demographic and clinical characteristics of the 95 patients with delirium based on the DSM- 5 criteria

\begin{tabular}{lc}
\hline \multicolumn{1}{c}{ Variable } & Total $(\mathrm{N}=95)$ \\
\hline Age (years), mean \pm SD & $77.58 \pm 7.26$ \\
Sex (male), N (\%) & $55(57.9)$ \\
Hyperactive type, N (\%) & $34(35.8)$ \\
Hypoactive type, N (\%) & $25(26.3)$ \\
Mixed type, N (\%) & $30(31.6)$ \\
No subtype, N (\%) & $6(6.3)$ \\
Number of medications received, mean \pm SD & $5.81 \pm 3.13$ \\
Comorbid cognitive impairment, N (\%) & $17(17.9)$ \\
NIHSS score at admission, mean $\pm S D$ & $5.41 \pm 4.50$ \\
Mean K-DRS-98 severity score, mean \pm SD & $18.39 \pm 4.13$ \\
Duration of delirium (days), mean \pm SD & $7.10 \pm 6.36$
\end{tabular}

SD: standard deviation, NIHSS: National Institutes of Health Stroke Scale, K-DRS-98: the Korean version of the Delirium Rating ScaleRevised-98

dition, the mRS score on admission was significantly higher in the hypoactive subtype group $(3.96 \pm 1.24)$ than in the other subtype groups $(\mathrm{p}<0.01)$.

\section{Outcomes and motor subtypes}

Table 3 shows the outcomes according to the motor subtype of delirium. The duration of delirium was significantly longer in the mixed subtype group (9.96 \pm 7.29 days) than in the other subtype groups $(\mathrm{p}<0.01)$. Although the mean KDRS-98 severity score over the course of the delirium episode was higher in the mixed subtype group (19.27 \pm 4.94$)$ than in the other subtype groups, the difference was not significant $(\mathrm{p}=0.57)$. However, the highest K-DRS-98 severity score over the course of the delirium episode was significantly higher in the mixed subtype group (22.46 \pm 5.33$)$ than the other subtype groups $(\mathrm{p}=0.03)$. The hypoactive subtype group $(36.88 \pm 27.71)$ had a significantly longer hospital stay than the other subtype groups $(\mathrm{p}<0.01)$ as well as the highest NIHSS $(6.36 \pm 5.42, \mathrm{p}=$ $0.03)$ and $m R S$ scores $(3.44 \pm 1.55, \mathrm{p}<0.01)$ at discharge. However, the groups did not significantly differ in terms of institutionalization at discharge and death during hospitalization.

The results of the multiple linear regression analysis for the outcome variables are shown in Table 4. Motor subtype of delirium was independently associated with the duration of delirium and the highest K-DRS-98 severity score. Especially the hypoactive and mixed subtypes had a greater effect on longer duration and severe delirium symptoms than the hyperactive subtype. In temrs of length of hospital stay, the variables that predicted long-term hospitalization were motor subtype and $\mathrm{mRS}$ score at admission. Additionally, the hypoactive subtype had a greater effect on length of hospital stay than the hyper- 
active subtype. After adjusting for variables, none of the motor subtypes of delirium were associated with NIHSS and mRS scores at discharge.

\section{DISCUSSION}

This study examined the relationship between the delirium motor subtypes and the clinical characteristics and outcomes

Table 2. Clinical characteristics and motor subtypes of the 95 patients with delirium

\begin{tabular}{|c|c|c|c|c|c|}
\hline Variable & Hyperactive $(\mathrm{N}=34)$ & Hypoactive (N=25) & Mixed $(\mathrm{N}=30)$ & No subtype $(\mathrm{N}=6)$ & $\mathrm{p}$ \\
\hline Sex (male), N (\%) & $22(64.7)$ & $14(56.0)$ & $18(60.0)$ & $1(16.7)$ & 0.16 \\
\hline Age (years), mean $\pm \mathrm{SD}$ & $78.76 \pm 5.50$ & $76.76 \pm 8.78$ & $77.36 \pm 7.39$ & $75.33 \pm 9.29$ & 0.90 \\
\hline $\mathrm{BUN}(\mathrm{mg} / \mathrm{dL})$, mean $\pm \mathrm{SD}$ & $16.04 \pm 5.23$ & $17.96 \pm 5.89$ & $21.05 \pm 8.53$ & $18.07 \pm 6.89$ & 0.03 \\
\hline Number of medications received, mean $\pm \mathrm{SD}$ & $5.20 \pm 2.70$ & $6.08 \pm 3.39$ & $5.96 \pm 3.27$ & $7.33 \pm 3.61$ & 0.33 \\
\hline Comorbid cognitive impairment, $\mathrm{N}(\%)$ & $5(14.7)$ & $3(12.0)$ & $7(23.3)$ & $2(33.3)$ & 0.21 \\
\hline History of delirium, N (\%) & $4(11.8)$ & $2(8.0)$ & $6(20.0)$ & $0(0.0)$ & 0.87 \\
\hline \multicolumn{6}{|l|}{ Lesion, side } \\
\hline Left hemisphere, $\mathrm{N}(\%)$ & $8(23.5)$ & $7(28.0)$ & $11(36.7)$ & $3(50.0)$ & \\
\hline Right hemisphere, N (\%) & $18(52.9)$ & $13(52.0)$ & $13(43.3)$ & $3(50.0)$ & 0.13 \\
\hline Both hemisphere, $\mathrm{N}(\%)$ & $8(23.5)$ & $5(20.0)$ & $6(20.0)$ & $0(0.0)$ & \\
\hline NIHSS score on admission, mean \pm SD & $3.97 \pm 3.42$ & $6.72 \pm 4.75$ & $6.53 \pm 5.10$ & $2.50 \pm 1.87$ & 0.02 \\
\hline mRS score on admission, mean \pm SD & $2.44 \pm 1.52$ & $3.96 \pm 1.24$ & $3.33 \pm 1.29$ & $2.33 \pm 1.03$ & $<0.01$ \\
\hline
\end{tabular}

SD: standard deviation, BUN: blood urea nitrogen, NIHSS: National Institutes of Health Stroke Scale, mRS: modified Rankin Scale

Table 3. Motor subtypes and outcomes in the 95 patients with delirium

\begin{tabular}{|c|c|c|c|c|c|}
\hline Variable & Hyperactive $(\mathrm{N}=34)$ & Hypoactive $(\mathrm{N}=25)$ & Mixed $(\mathrm{N}=30)$ & No subtype $(\mathrm{N}=6)$ & $\mathrm{P}$ \\
\hline Duration of delirium (days), mean $\pm S D$ & $4.17 \pm 3.96$ & $8.68 \pm 6.49$ & $9.96 \pm 7.29$ & $2.83 \pm 2.31$ & $<0.01$ \\
\hline Mean K-DRS-98 severity, mean \pm SD & $17.83 \pm 4.17$ & $18.43 \pm 3.91$ & $19.27 \pm 4.94$ & $17.00 \pm 2.60$ & 0.57 \\
\hline Highest K-DRS-98 severity, mean \pm SD & $19.09 \pm 4.53$ & $21.32 \pm 5.14$ & $22.46 \pm 5.33$ & $18.00 \pm 4.00$ & 0.03 \\
\hline Length of stay (days), mean \pm SD & $15.14 \pm 14.71$ & $36.88 \pm 27.71$ & $21.90 \pm 19.10$ & $13.83 \pm 16.83$ & $<0.01$ \\
\hline Institutionalization, $\mathrm{N}(\%)$ & $11(32.4)$ & $16(66.7)$ & $12(41.4)$ & $1(16.7)$ & 1.00 \\
\hline Died during hospitalization, N (\%) & $0(0.0)$ & $1(4.0)$ & $1(3.3)$ & $0(0.0)$ & 0.70 \\
\hline NIHSS score at discharge, mean \pm SD & $3.17 \pm 4.53$ & $6.36 \pm 5.42$ & $3.66 \pm 3.31$ & $1.83 \pm 2.56$ & 0.03 \\
\hline mRS score at discharge, mean $\pm S D$ & $2.00 \pm 1.67$ & $3.44 \pm 1.55$ & $3.00 \pm 1.62$ & $1.66 \pm 1.36$ & $<0.01$ \\
\hline
\end{tabular}

SD: standard deviation, K-DRS-98: the Korean version of the Delirium Rating Scale-Revised-98, NIHSS: National Institutes of Health Stroke Scale, mRS: modified Rankin Scale

Table 4. Predictors of outcome variables in the 95 patients with delirium (multiple linear regression analysis)

\begin{tabular}{|c|c|c|c|c|c|c|c|c|c|c|}
\hline & \multicolumn{2}{|c|}{$\begin{array}{l}\text { Duration of } \\
\text { delirium }\end{array}$} & \multicolumn{2}{|c|}{$\begin{array}{l}\text { Highest K-DRS-98 } \\
\text { severity }\end{array}$} & \multicolumn{2}{|c|}{ Length of stay } & \multicolumn{2}{|c|}{$\begin{array}{c}\text { NIHSS } \\
\text { at discharge }\end{array}$} & \multicolumn{2}{|c|}{$\begin{array}{l}\mathrm{mRS} \\
\text { at discharge }\end{array}$} \\
\hline & $\mathrm{B}$ & $\mathrm{P}$ & $\mathrm{B}$ & $\mathrm{P}$ & $\mathrm{B}$ & $\mathrm{P}$ & $\mathrm{B}$ & $\mathrm{P}$ & $\mathrm{B}$ & $\mathrm{P}$ \\
\hline NIHSS at admission & -0.05 & 0.72 & -0.10 & 0.45 & -0.28 & 0.59 & 0.33 & $<0.01$ & 0.02 & 0.54 \\
\hline mRS at admission & 0.79 & 0.10 & -0.16 & 0.69 & 4.25 & 0.01 & 0.34 & 0.31 & 0.44 & $<0.01$ \\
\hline BUN & -0.03 & 0.28 & -0.03 & 0.21 & -0.08 & 0.40 & -0.02 & 0.25 & -0.01 & 0.15 \\
\hline \multicolumn{11}{|l|}{ Motor subtypes } \\
\hline (Hypoactive, hyperactive) & 3.51 & 0.04 & 2.81 & 0.05 & 16.21 & $<0.01$ & 1.78 & 0.14 & 0.71 & 0.10 \\
\hline (Mixed, hyperactive) & 5.58 & 0.01 & 4.13 & 0.02 & 4.63 & 0.38 & -0.41 & 0.71 & 0.66 & 0.10 \\
\hline (No subtype, hyperactive) & -1.37 & 0.59 & -1.28 & 0.56 & -1.35 & 0.87 & -0.83 & 0.65 & -0.26 & 0.69 \\
\hline
\end{tabular}

The motor subtype of delirium was represented as three dummy variables with the hyperactive subtype serving as the reference group. B: unstandardized coefficient, NIHSS: National Institutes of Health Stroke Scale, mRS: modified Rankin Scale, BUN: blood urea nitrogen, K-DRS-98: the Korean version of the Delirium Rating Scale-Revised-98 
in ischemic stroke patients. The incidence of delirium in the present study $(10.18 \%)$ was in the low range of reported results $(2.3-61 \%) .^{9}$ A recent study ${ }^{32}$ conducted in a primary, national certified SU found that the incidence of delirium is $10.7 \%$, which is similar to the present results even though they were derived from all stroke patients, including those with ischemic and hemorrhagic infarcts. This finding may be due to the improvements in stroke care that have developed over the last several years. ${ }^{33}$ In particular, our hospital operates as a Regional Comprehensive Stroke Center at which the detection and intervention of cerebral infarction is relatively early due to the rapid initial brain imaging scans and the execution of thrombolysis, ${ }^{34}$ which could reduce the incidence of delirium.

Of the delirium patients, $35.8 \%$ had the hyperactive subtype, followed by the mixed subtype (31.6\%), the hypoactive subtype (26.3\%), and no subtype (6.3\%). Previous studies of post-stroke delirium ${ }^{32,35}$ revealed that the mixed and hypoactive subtypes were the most common, whereas the hyperactive subtype was the most common in our study. A possible explanation for this difference is that many patients with coma and global or sensory aphasia could simultaneously have hypoactive delirium, and as many of these patients were excluded from the study, the number of hypoactive delirium patients may have been underestimated. Although this may be a limitation of the present study, it was no possible to include these patients in the analyses because their motor profiles and nonmotor symptoms of delirium could not be adequately assessed.

In studies ${ }^{36-38}$ investigating the relationship between etiology and motor subtype, there may be a dominant motor subtype for each of the different types of delirium, attributable to a certain etiology. On the other hand, Sagawa et al. ${ }^{39}$ found no association between etiology and motor subtype in a crosssectional assessment of 100 cancer patients. Similarly, we did not find major associations between motor subtype and etiology, but these relationships may differ by study population and methodology. We identified delirium patients based on daily screening and assessment of all SU inpatients, while the other studies relied on psychiatry consultation/liaison services for identifying delirium subjects. In addition, our study was prospectively designed, whereas other studies retrospectively evaluated delirium patients.

The NIHSS and mRS scores on admission were the highest in the hypoactive subtype groups among all of the motor subtype groups. This suggests that the incidence of hypoactive delirium may be higher in patients with more severe stroke symptoms and poorer physical function. Several studies ${ }^{17,36,39}$ have examined the relationships between factors affecting delirium and motor subtype in patients with various medical conditions that cause delirium. Although one study ${ }^{21}$ investigated the relationships between delirium motor subtype and factors such as stroke etiology, lesion location, and severity of symptoms in stroke patients, including both ischemic and hemorrhagic infarcts, to our knowledge, ours is the first study to assess ischemic stroke only. In addition, most studies have been limited by their cross-sectional design and lack of control of confounding factors, such as medication exposure, age, and comorbid dementia status, as was also the case in the present study. Therefore, much larger studies may be needed to clarify the relationship between delirium motor subtypes and strokerelated factors, such as symptoms and lesion location and size.

Meagher et al. ${ }^{17}$ found that the mixed subtype of delirium is associated with significantly more severe symptoms and a higher mean dose of antipsychotics than any other motor subtypes and that patients with hypoactive delirium are more likely to die within 30 days of study entry than those with other delirium subtypes. However, other than the above report, no studies have classified and compared the motor subtypes of delirium using validated instruments. In this study, patients with the hypoactive and mixed subtypes of delirium tended to have poorer prognoses that were reflected by a longer duration of delirium, more severe symptoms of delirium, and a longer hospital stay than those with the hyperactive or no subtype of delirium. These poorer outcomes may be attributable to many factors, including the confounding effects of older age, more frequent comorbid dementia, ${ }^{19}$ greater delays in detection, ${ }^{40,41}$ more prolonged delirium, less frequent use of pharmacotherapies, and differences in the frequency and type of complications (e.g., higher rates of pressure sores and aspiration pneumonia). Although it was not possible to consider all possible confounding factors in the present study, the patients with delirium who exhibited hypoactive motor elements had worse outcomes after adjusting for variables that showed significant differences between motor subtypes. Previous studies in patients with various medical conditions ${ }^{15,42}$ have reported poorer prognoses and fewer reversible symptoms in hypoactive patients, which is similar to our findings.

The assessment of delirium is often difficult and many cases may be missed, especially in stroke patients, due to the high prevalence of language disorders, neglect, and mood disturbances that can be confused with delirium. Therefore, systematic assessments and longitudinal observations by medical personnel will be necessary to yield reliable data. In our study, assessments were conducted every day using screening methods with high sensitivity and specificity, and the final diagnosis was based on a daily observational chart provided by medical personnel.

The present study has several limitations that should be considered. First, we performed only a cross-sectional evaluation of the clinical characteristics and symptoms of patients with different motor subtypes of delirium. However, delirium 
is a complex neuropsychiatric syndrome in which a fluctuating course is a key characteristic in both the DSM and International Classification of Diseases (ICD) diagnostic systems. ${ }^{1,43}$ This variability means that the phenomenological profile of delirium is best captured by longitudinal studies that assess symptoms over a sustained period. Therefore, a systematic and large-scale study will be needed to assess delirium symptoms and motor subtypes longitudinally while also considering factors that may influence the course of delirium, such as medication use, infection, and metabolic problems. Second, in patients who were discharged with still-unresolved delirium, the duration of delirium and the severity of symptoms were assessed by means of follow-up calls or visits, which may be less accurate than formal assessments performed during hospitalization. Third, preexisting mild cognitive impairment or dementia was identified only through histories provided by patients and their caregivers. The lack of objective and accurate assessment of baseline cognitive functioning is a limitation when evaluating cognitive decline; thus, future studies should include methods to assess cognitive function before delirium develops, such as the Informant Questionnaire on Cognitive Decline in the Elderly (IQCODE), ${ }^{44}$ so that outcomes associated with cognitive function can be determined according to delirium motor subtypes.

In conclusion, the present results suggest that motor subtype of delirium is associated with different characteristics and outcomes in ischemic stroke patients. In particular, the patients with delirium that included elements of the hypoactive motor profile were associated with a greater severe stroke-related disability and poorer outcomes than those without it.

\section{Conflicts of Interest}

The authors have no potential conflicts of interest to disclose.

\section{Author Contributions}

Conceptualization: Hee Won Yang, Jeong Lan Kim. Data curation: Miji Lee, Jong Wook Shin. Formal analysis: Hee Won Yang, Jeong Lan Kim. Funding acquisition: Jei Kim, Jeong Lan Kim. Investigation: Hee Won Yang, Jong Wook Shin, Hye Seon Jeong, Jei Kim, Jeong Lan Kim. Methodology: Hee Won Yang, Jei Kim, Jong Wook Shin, Jeong Lan Kim. Project administration: Hee Won Yang, Hye Seon Jeong, Jei Kim, Jeong Lan Kim. Resources: Jei Kim, Jeong Lan Kim. Software: Hee Won Yang, Jong Wook Shin. Supervision: Jei Kim, Jeong Lan Kim. Validation: Hee Won Yang, Jeong Lan Kim, Hye Seon Jeong. Visualization: Hee Won Yang, Jeong Lan Kim. Writingoriginal draft: Hee Won Yang, Jeong Lan Kim. Writing_review \& editing: Jeong Lan Kim, Jei Kim, Hee Won Yang.

\section{ORCID iDs}

$\begin{array}{ll}\text { Jeong Lan Kim } & \text { https://orcid.org/0000-0002-6554-4637 } \\ \text { Hee Won Yang } & \text { https://orcid.org/0000-0002-2460-1869 }\end{array}$

\section{REFERENCES}

1. American Psychiatric Association. Diagnostic and Statistical Manual of Mental Disorders $\left(D S M-5^{\circledR}\right)$. Washington DC: American Psychiatric
Pub; 2013.

2. Witlox J, Eurelings LS, de Jonghe JF, Kalisvaart KJ, Eikelenboom P, van Gool WA. Delirium in elderly patients and the risk of postdischarge mortality, institutionalization, and dementia: a meta-analysis. JAMA 2010; 304:443-451.

3. Guerini F, Frisoni GB, Morghen S, Speciale S, Bellelli G, Trabucchi M. Clinical instability as a predictor of negative outcomes among elderly patients admitted to a rehabilitation ward. J Am Med Dir Assoc 2010;11: 443-448.

4. Morandi A, McCurley J, Vasilevskis EE, Fick DM, Bellelli G, Lee P, et al. Tools to detect delirium superimposed on dementia: a systematic review. J Am Geriatr Soc 2012;60:2005-2013.

5. Shi Q, Presutti R, Selchen D, Saposnik G. Delirium in acute stroke: a systematic review and meta-analysis. Stroke 2012;43:645-649.

6. Oldenbeuving AW, de Kort PL, Jansen BP, Roks G, Kappelle LJ. Delirium in acute stroke: a review. Int J Stroke 2007;2:270-275.

7. van Rijsbergen MW, Oldenbeuving AW, Nieuwenhuis-Mark RE, Nys GM, Las SG, Roks G, et al. Delirium in acute stroke: a predictor of subsequent cognitive impairment? A two-year follow-up study. J Neurol Sci 2011;306:138-142.

8. Indredavik B, Rohweder G, Naalsund E, Lydersen S. Medical complications in a comprehensive stroke unit and an early supported discharge service. Stroke 2008;39:414-420.

9. Elzbieta K, Tomasz D, Katarzyna K, Agnieszka S, Aleksandra K. Knowns and unknowns about delirium in stroke: a review. Cogn Behav Neurol 2016;29:174-189.

10. Oldenbeuving AW, de Kort PL, Jansen BP, Algra A, Kappelle LJ, Roks G. Delirium in the acute phase after stroke: incidence, risk factors, and outcome. Neurology 2011;76:993-999.

11. Carin-Levy G, Mead GE, Nicol K, Rush R, van Wijck F. Delirium in acute stroke: screening tools, incidence rates and predictors: a systematic review. J Neurol 2012;259:1590-1599.

12. Lipowski ZJ. Delirium in the elderly patient. N Engl J Med 1989;320:578582.

13. Liptzin B, Levkoff SE. An empirical study of delirium subtypes. Br J Psychiatry 1992;161:843-845.

14. Meagher DJ, Moran M, Raju B, Gibbons D, Donnelly S, Saunders J, et al. Motor symptoms in 100 patients with delirium versus control subjects: comparison of subtyping methods. Psychosomatics 2008;49:300308.

15. Meagher D, Moran M, Raju B, Leonard M, Donnelly S, Saunders J, et al. A new data-based motor subtype schema for delirium. J Neuropsychiatry Clin Neurosci 2008;20:185-193.

16. Godfrey A, Leonard M, Donnelly S, Conroy M, Olaighin G, Meagher D. Validating a new clinical subtyping scheme for delirium with electronic motion analysis. Psychiatry Res 2010;178:186-190.

17. Meagher DJ, Leonard M, Donnelly S, Conroy M, Adamis D, Trzepacz PT. A longitudinal study of motor subtypes in delirium: relationship with other phenomenology, etiology, medication exposure and prognosis. J Psychosom Res 2011;71:395-403.

18. Marcantonio E, Ta T, Duthie E, Resnick NM. Delirium severity and psychomotor types: their relationship with outcomes after hip fracture repair. J Am Geriatr Soc 2002;50:850-857.

19. Kiely DK JR, Bergmann MA, Marcantonio ER. Association between psychomotor activity delirium subtypes and mortality among newly admitted postacute facility patients. J Gerontol 2007;62A:174-179.

20. Kobayashi K TO, Suzuki M, Yamaguchi N. A retrospective study of delirium subtype. Jpn J Psychiatry Neurol 1992;46:911-917.

21. Pasinska P, Kowalska K, Klimiec E, Wilk A, Szyper-Maciejowska A, Dziedzic T, et al. Poststroke Delirium Clinical Motor Subtypes: The PRospective Observational POLIsh Study (PROPOLIS). J Neuropsychiatry Clin Neurosci 2019;31:104-111.

22. Qu J, Chen Y, Luo G, Zhong H, Xiao W, Yin H. Delirium in the acute phase of ischemic stroke: incidence, risk factors, and effects on functional outcome. J Stroke Cerebrovasc Dis 2018;27:2641-2647. 
23. Klimiec-Moskal E, Lis A, Pera J, Slowik A, Dziedzic T. Subsyndromal delirium is associated with poor functional outcome after ischaemic stroke. Eur J Neurol 2019;26:927-934.

24. Trzepacz PT, Mittal D, Torres R, Kanary K, Norton J, Jimerson N. Validation of the Delirium Rating Scale-revised-98: comparison with the delirium rating scale and the cognitive test for delirium. J Neuropsychiatry Clin Neurosci 2001;13:229-242.

25. Inouye SK, van Dyck CH, Alessi CA, Balkin S, Siegal AP, Horwitz RI. Clarifying confusion: the confusion assessment method. A new method for detection of delirium. Ann Intern Med 1990;113:941-948.

26. Shi Q, Warren L, Saposnik G, Macdermid JC. Confusion assessment method: a systematic review and meta-analysis of diagnostic accuracy. Neuropsychiatr Dis Treat 2013;9:1359-1370.

27. Lim KO, Kim SY, Lee YH, Lee SW, Kim JL. A Validation Study for the Korean Version of Delirium Rating Scale-Revised-98 (K-DRS-98). J Korean Neuropsychiatr Assoc 2006;45:518-526.

28. Kim HK, Lee MJ, Kwon IS, Kim JL. Validation of the Korean version of the delirium motor subtype scale. Psychiatry Investig 2018;15:6-12.

29. Adams HP Jr, Bendixen BH, Kappelle LJ, Biller J, Love BB, Gordon DL, et al. Classification of subtype of acute ischemic stroke. Definitions for use in a multicenter clinical trial. TOAST. Trial of Org 10172 in Acute Stroke Treatment. Stroke 1993;24:35-41.

30. Meyer BC, Lyden PD. The modified National Institutes of Health Stroke Scale: its time has come. Int J Stroke 2009;4:267-273.

31. Quinn TJ, Dawson J, Walters MR, Lees KR. Reliability of the modified Rankin Scale: a systematic review. Stroke 2009;40:3393-3395.

32. Nydahl P, Bartoszek G, Binder A, Paschen L, Margraf NG, Witt K, et al. Prevalence for delirium in stroke patients: a prospective controlled study. Brain Behav 2017;7:e00748.

33. Dahl MH, Ronning OM, Thommessen B. Delirium in acute stroke-prevalence and risk factors. Acta Neurol Scand Suppl 2010;(190):3943.
34. Kim J, Hwang YH, Kim JT, Choi NC, Kang SY, Cha JK, et al. Establishment of government-initiated comprehensive stroke centers for acute ischemic stroke management in South Korea. Stroke 2014;45:2391-2396.

35. Pasinska P, Kowalska K, Klimiec E, Szyper-Maciejowska A, Wilk A, Klimkowicz-Mrowiec A. Frequency and predictors of post-stroke delirium in PRospective Observational POLIsh Study (PROPOLIS). J Neurol 2018;265:863-870.

36. Gupta AK, Saravay SM, Trzepacz PT, Chirayu P. Delirium motoric subtypes. Psychosomatics 2005;46:158.

37. Meagher DJ, Moran M, Raju B, Gibbons D, Donnelly S, Saunders J, et al. Phenomenology of delirium. Assessment of 100 adult cases using standardised measures. Br J Psychiatry 2007;190:135-141.

38. Olofsson SM, Weitzner MA, Valentine AD, Baile WF, Meyers CA. A retrospective study of the psychiatric management and outcome of delirium in the cancer patient. Support Care Cancer 1996;4:351-357.

39. Sagawa R, Akechi T, Okuyama T, Uchida M, Furukawa TA. Etiologies of delirium and their relationship to reversibility and motor subtype in cancer patients. Jpn J Clin Oncol 2009;39:175-182.

40. Inouye SK, Foreman MD, Mion LC, Katz KH, Cooney LM Jr. Nurses' recognition of delirium and its symptoms: comparison of nurse and researcher ratings. Arch Intern Med 2001;161:2467-2473.

41. Han JH, Zimmerman EE, Cutler N, Schnelle J, Morandi A, Dittus RS, et al. Delirium in older emergency department patients: recognition, risk factors, and psychomotor subtypes. Acad Emerg Med 2009;16: 193-200.

42. Lam PT, Tse CY, Lee CH. Delirium in a palliative care unit. Prog Palliat Care 2003;11:126-133.

43. WHO. The ICD-10 Classification of Mental and Behavioral Disorders. Geneva: WHO; 1993.

44. Jorm AF. The Informant Questionnaire on cognitive decline in the elderly (IQCODE): a review. Int Psychogeriatr 2004;16:275-293. 